LBNL-52390

Paper submitted to the International Conference on Ion Sources (ICIS'03)

Dubna, Russia, September 6-12, 2003

and to be published in Rev. Sci. Instrum. as part of the Proceedings.

\title{
MEVVA ION SOURCE OPERATED IN PURELY GASEOUS MODE*
}

Gera Yu. Yushkov ${ }^{1}$, Robert A. MacGill ${ }^{2}$ and Ian G. Brown ${ }^{2}$

${ }^{1}$ High Current Electronics Institute, Russian Academy of Sciences, Tomsk 634050, Russia

${ }^{2}$ Lawrence Berkeley National Laboratory, Berkeley, CA 94720

March 2003

* Supported by the U.S. Department of Energy's Office of Energy Research and the Initiatives for Proliferation Prevention (IPP) Program under Contract Number DE-AC03-76SF00098. 


\title{
Mevva Ion Source Operated in Purely Gaseous Mode
}

Gera Yu. Yushkov ${ }^{\text {a) }}$

High Current Electronics Institute, Russian Academy of Sciences, Tomsk 634050, Russia

Robert A. MacGill and lan G. Brown

Lawrence Berkeley National Laboratory, 1 Cyclotron Road, Berkeley, CA 94720

\begin{abstract}
$\underline{\text { Abstract }}$
We have operated a vacuum arc ion source in such a way as to form beams of purely gaseous ions. The vacuum arc configuration that is conventionally used to produce intense beams of metal ions was altered so as to form gaseous ion beams, with only minimal changes to the external circuitry and no changes at all internally to the ion source. In our experiments we formed beams from oxygen $\left(\mathrm{O}^{+}\right.$and $\left.\mathrm{O}_{2}^{+}\right)$, nitrogen $\left(\mathrm{N}^{+}\right.$and $\left.\mathrm{N}_{2}{ }^{+}\right)$, argon $\left(\mathrm{Ar}^{+}\right)$and carbon dioxide $\left(\mathrm{C}^{+}, \mathrm{CO}_{2}{ }^{+}, \mathrm{O}^{+}\right.$and $\left.\mathrm{O}_{2}^{+}\right)$at extraction voltage of 2 to $50 \mathrm{kV}$. We used a pulsed mode of operation, with beam pulses approximately 50 milliseconds long and repetition rate 10 pulses per second, for a duty cycle of about $50 \%$. Downstream ion beam current as measured by a $5 \mathrm{~cm}$ diameter Faraday cup was typically $0.5 \mathrm{~mA}$ pulse or about $250 \mu \mathrm{A}$ time averaged. This time averaged beam current is very similar to that obtained for metal ions when the source is operated in the usual vacuum arc mode. Here we describe the modifications made to the source and the results of our investigations.
\end{abstract}

a) Electronic mail: gyushkov@opee.hcei.tsc.ru 


\section{INTRODUCTION}

Vacuum arc ion sources are an established and versatile tool for forming high current metal ion beams [1-3]. In the usual mode of operation of these sources the beam contains virtually all metal ions, with only minimal gaseous ion impurity content. It has been shown that gas can be deliberately introduced into the vacuum arc plasma discharge so as to form beams containing controllable mixtures of metal and gaseous ion species [4,5]. Hybrid metal-gaseous ion beams can be advantageous for some metallurgical ion implantation applications, for example for forming buried oxide layers [5]. Although the metal-gas ion ratio can be varied over a wide range by this method, it is not possible to reduce the metal ion content to less that several percent, and thus beams of purely gaseous ion species cannot be formed. It would be experimentally convenient if it were possible to use a vacuum arc ion source not only to form metal ion beams and mixed metal-gas ion beams, but also to form beams containing essentially $100 \%$ gaseous ions. One would then have a single ion source for producing ions beams of all of the elements of the Periodic Table. We have developed such a method. Requiring only minor reconfiguration of the external electrical system and no changes at all to the ion source hardware, the technique allows quick and simple change-over from metal-ion or hybrid mode to purely gaseous-ion mode. Here we describe the ion source system setup that we've used and outline the results that we've obtained that exemplify purely gaseous mode operation.

\section{DESCRIPTION OF THE ION SOURCE AND EXPERIMENTAL SETUP}

Our approach to forming purely gaseous ion beams was to replace the vacuum arc as plasma formation mechanism by a gaseous plasma that is a type of hollow cathode glow discharge, while still utilizing the same geometry and mechanical configuration as for the metal vapor arc. The work was carried out at the Lawrence Berkeley National Laboratory using the 
Mevva $\mathrm{V}$ ion source that has been fully described elsewhere [1,6,7]. A simplified schematic of the ion source and its electrical system is shown in Figure 1. Only three small modifications were needed: (i) a gas inlet feed was utilized (this change had been made previously as part of earlier hybrid metal-gas ion beam work [5]; the optional gas inlet is a permanent part of the Mevva source); (ii) the polarity of the trigger pulse and of the arc power supply (the LC pulse forming line as used for the vacuum arc configuration) was reversed; this needed no more than switching around the leads; (iii) installation of a current-limiting high power resistor of 1 to 3 $\mathrm{k} \Omega$ in the plasma discharge circuit.

The source was mounted to a vacuum chamber as used normally for Mevva-V ion source experimental work [1,7]. Base vacuum pressure was about $1 \times 10^{-6}$ Torr, and in operation gas was fed into the source such that the chamber pressure was about 1 to $2 \times 10^{-4}$ Torr. A magnetically-suppressed Faraday cup with $5 \mathrm{~cm}$ diameter entrance aperture was located $65 \mathrm{~cm}$ downstream from the ion source extractor, and was used to monitor the ion beam current. The Faraday cup was radially moveable and could be retracted completely from the ion beam path so as to allow operation of a time-of-flight (TOF) charge state analysis system $[1,8,9]$ by means of which the ion beam charge-to-mass composition was measured.

\section{GASEOUS SOURCE AND BEAM CHARACTERISTICS}

When the source is triggered by a high voltage $(\sim 3 \mathrm{kV})$, high current $(\sim 5 \mathrm{~A})$, negative pulse applied between the trigger electrode and the anode (where now the anode is what was previously the vacuum arc cathode), a hollow cathode glow discharge plasma [10-12] is formed in the cavity that now includes a hollow cathode and the extractor grid at cathode potential. As is usual for low pressure glow discharges with anode-cathode surface area ratio $\sim\left(\mathrm{m}_{\mathrm{e}} / \mathrm{M}_{\mathrm{i}}\right)^{1 / 2}$, most of the discharge voltage is located at the negative cathode drop. Plasma ions accelerated by this 
potential bombard the cathode surface and produce secondary electrons, which are then accelerated into the plasma volume by the cathode potential drop. The electrons oscillate within the hollow-cathode plasma configuration, establishing an efficient ionization mechanism in the gas. The thickness $d_{c}$ of the cathode fall region may be estimated from the Child-Langmuir equation as:

$$
d_{c}=2 / 3\left[\varepsilon_{0}\left(2 e / M_{i}\right)^{1 / 2}\left(\phi_{c}{ }^{3 / 2} / I_{d}\right) S_{c}(1+\gamma)\right]^{1 / 2}
$$

where $\phi_{c}$ is the cathode potential drop, $I_{d}$ is the discharge current, $S_{c}$ is the hollow cathode surface area, and $\gamma$ is the secondary electron emission coefficient. For typical discharge conditions in the present work, $\phi_{c} \approx 500 \mathrm{~V}, I_{d} \approx 0.5 \mathrm{~A}, S_{c} \approx 700 \mathrm{~cm}^{2}$, and $\gamma \approx 0.1$, and the thickness $d_{c}$ of the cathode fall region determined from Eq. (1) is about $0.5 \mathrm{~cm}$. On the other hand, the ionization mean-free-path for energetic electrons within the hollow cathode region is

$$
\lambda=1 /\left(3.3 \times 10^{16} P \sigma_{i}\right),
$$

where $P$ is the gas pressure and $\sigma_{i}$ is the cross section for electron impact ionization. For our experimental condition $\left(P \approx 2 \times 10^{-4}\right.$ Torr and $\left.\sigma_{i} \approx 10^{-16} \mathrm{~cm}^{2}\right), \lambda$ is about $15 \mathrm{~m}$. Since $\lambda>>d_{c}$ (and also $\lambda>>l_{c}$, where $l_{c} \approx 10 \mathrm{~cm}$ is the characteristic length of the hollow-cathode cavity), the electrons execute many $\left(>10^{2}\right)$ oscillations between ionizing collisions. Thus, finally, the probability of ionization is uniform throughout the volume, and a uniform density plasma of gaseous ions is formed by the reflexing electrons.

The series current-limiting resistor that was installed in the discharge power supply circuit determines the current and also extends the pulse length. In vacuum arc mode, when the arc impedance is very low (a few tens of $\mathrm{m} \Omega$ ), the discharge current is typically $100-300 \mathrm{~A}$ and the pulse length, determined by the LC pulse line, is usually $250 \mu \mathrm{s}$. In gaseous mode (hollow cathode glow mode) the discharge current is typically about $0.5 \mathrm{~A}$ and the pulse length, now determined by the glow discharge extinction, is about $50 \mathrm{mS}$. Figure 2 shows the discharge voltage-current characteristics and Figure 3 shows a typical oscillogram of the discharge and 
beam current time histories. We point out that the ion beam noise $\left(\delta I_{\text {beam }} / I_{\text {beam }}\right)$ is quite low, of order $1 \%$ rms.

The beam current as measured by the downstream Faraday cup is shown in Figure 4 as a function of ion source extractor voltage. This is the pulse current (beam current measured when the beam pulse is on); for the $50 \%$ duty cycle used in the present work, the time-averaged beam current is one half of this value. The Faraday cup, being downstream $65 \mathrm{~cm}$ and having a $5 \mathrm{~cm}$ diameter entrance aperture, collects a current $\left(I_{F C}\right)$ that is a only a small fraction of the total ion beam current $\left(I_{t o t}\right)$ formed at the extractor; i.e., the beam is not a particularly low emittance beam. We estimate $I_{F C} / I_{t o t} \sim 0.01-0.1$. The electrical efficiency of the gaseous ion source can be written as $\alpha=I_{i o n} / I_{d}$, which we see is equal to about $10^{-2}-10^{-3}$. This compares favorably with the ratio $S_{\text {emiss }} / S_{\text {tot }}$, where $S_{\text {emiss }}$ is the area of the plasma within the ion source cavity that is presented to the extractor and $S_{\text {tot }}$ is the total surface area of the plasma within the ion source cavity.

A time-of-flight charge state spectrum of the ion beam, for the case of oxygen gas feed, is shown in Figure 5. The beam is composed of about one third singly ionized $\mathrm{O}^{+}$and two thirds singly ionized molecular $\mathrm{O}_{2}{ }^{+}$. In the work described here we did not attempt to maximize the atomic ion fraction. We also produced beams from nitrogen, argon and carbon dioxide, with the same accelerating voltage and having approximately the same beam current. We could not detect the presence of any metal ions (for example, from the cathode, anode or extractor grid materials) for this mode of operation, within the TOF instrumental resolution of $\sim 1 \%$. 


\section{CONCLUSION}

We have demonstrated the operation of a vacuum arc ion source in a purely gaseous ion beam mode. The changes to the vacuum arc ion source that are required to implement this mode of operation are quite simple and minimal, and the source can be changed in a matter of minutes from metal ion mode to gaseous ion mode without need for breaking vacuum. Beams of gaseous ions can be produced with pulsed current about $1 \mathrm{~mA}$, pulse width of order $50 \mathrm{~ms}$ and duty cycle about $50 \%$, corresponding to a time-averaged ion beam current of several hundred microamperes. This time-averaged ion current is essentially the same as that normally obtained when the source is used to form metal ion beams in vacuum arc mode. The beams are stable and reproducible. This development broadens the range of application of the vacuum arc ion source to include all of the elements of the Periodic Table, both gas and metal species.

\section{Acknowledgments}

This work was supported by the U.S. Department of Energy under Contract No. DE-AC0376SF00098. 


\section{$\underline{\text { References }}$}

1. I. G. Brown, Rev. Sci. Instrum. 65, 3061 (1994).

2. S. P. Bugaev, A. G. Nikolaev, E. M. Oks, P. M. Schanin and G. Yu. Yushkov, Rev. Sci. Instrum. 65, 3119 (1994).

3. I. G. Brown and E. M. Oks, IEEE Trans. Plasma Sci. 25, 1222 (1997).

4. A. N. Tyimentsev, Yu. P. Pinzhin, A. D. Korotaev, A. E. Behert, A. O. Savchenko, Yu. R. Kolobov, S. P. Bugaev, P. M. Schanin and G. Yu. Yushkov, Nucl. Instrum. Methods Phys. Res. B 80/81, 491 (1993).

5. E. M. Oks, G. Yu. Yushkov, P. J. Evans, A. Oztarhan, I. G. Brown, M. R. Dickinson, F. Liu, R. A. MacGill, O. R. Monteiro and Z. Wang, Nucl. Instrum. Methods Phys. Res. B 127/128, 782 (1997).

6. I. G. Brown, J. E. Galvin, R. A. MacGill and F. J. Paoloni, Rev. Sci. Instrum. 61, 577 (1990).

7. I. G. Brown, M. R. Dickinson, J. E. Galvin, X. Godechot and R. A. MacGill, Nucl. Instr. Methods Phys. Res. B 55, 506 (1991).

8. I. G. Brown, J. E. Galvin, R. A. MacGill and R. T. Wright, Rev. Sci. Instrum. 58, 1589 (1987).

9. I. G. Brown and X. Godechot, IEEE Trans. Plasma Sci. 19, 713 (1991).

10. E. M. Oks, A. V. Vizir and G. Yu. Yushkov, Rev. Sci. Instrum. 69, 853 (1998).

11. A. V. Vizir, G. Yu. Yushkov and E. M. Oks, Rev. Sci. Instrum. 71, 728 (2000).

12. N. V. Gavrilov, G. A. Mesyats, G. V Radkovski and V. V. Bersenev, Surf. Coat. Technol. 96, 81 (1997). 


\section{Figure Captions}

Fig. 1 Schematic of the Mevva ion source as configured to operate in gaseous mode.

Fig. 2 Voltage-current characteristics of the glow discharge formed in the ion source plasma cavity for oxygen and carbon dioxide gases.

Fig. 3 Typical oscillogram showing:

Upper trace: discharge current supporting the plasma glow $(0.2 \mathrm{~A} / \mathrm{cm})$

Lower trace: ion beam current measured by downstream Faraday cup (200 $\mu \mathrm{A} / \mathrm{cm})$

Sweep speed is $10 \mathrm{~ms} / \mathrm{cm}$.

Fig. 4 Ion beam current measured by a 5-cm diameter Faraday cup located $65 \mathrm{~cm}$ downstream from the ion source, as a function of ion source extraction voltage.

Fig. 5 Time-of-flight oscillogram showing the charge state distribution of oxygen ion beam. 


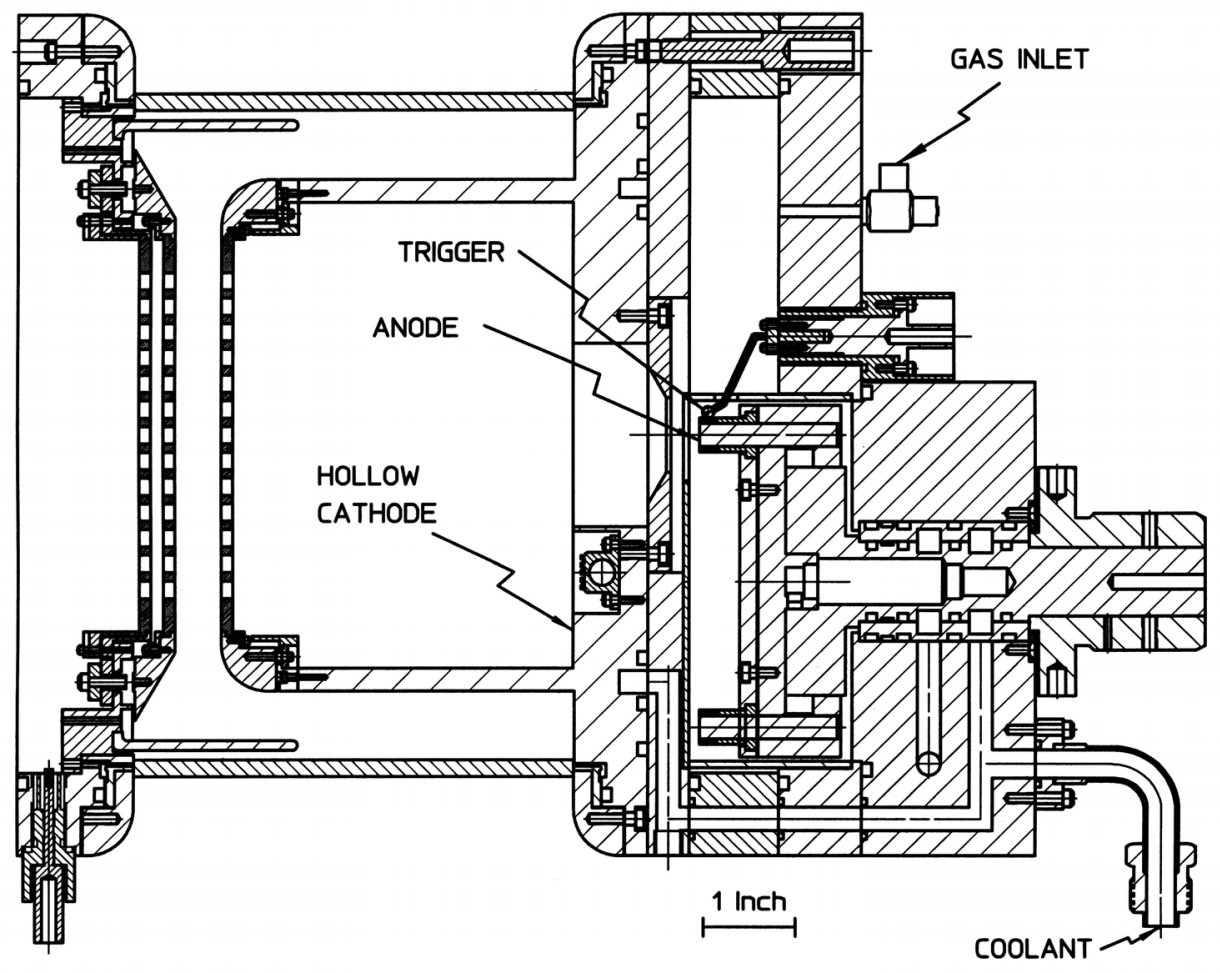

Figure 1 


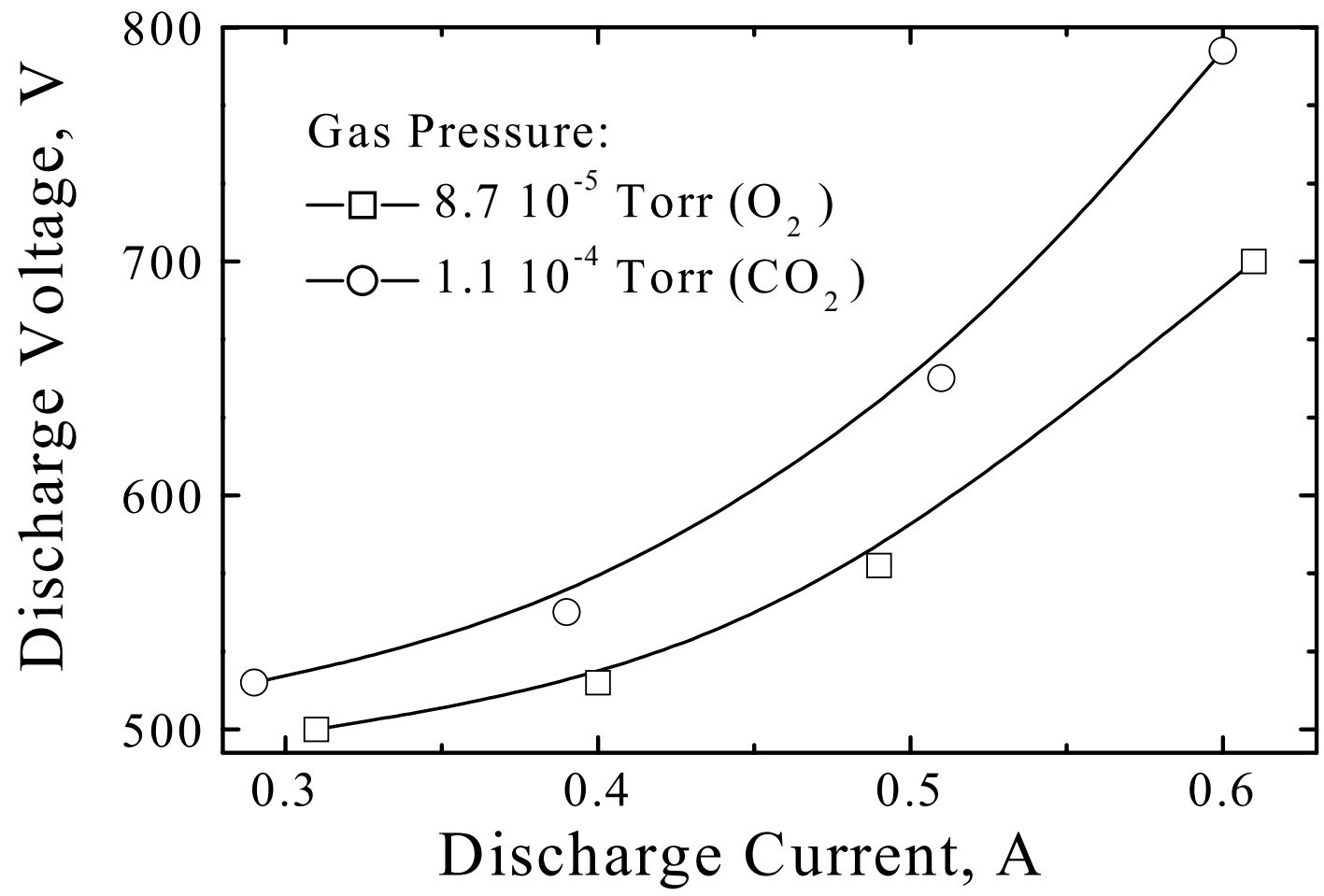

Figure 2 


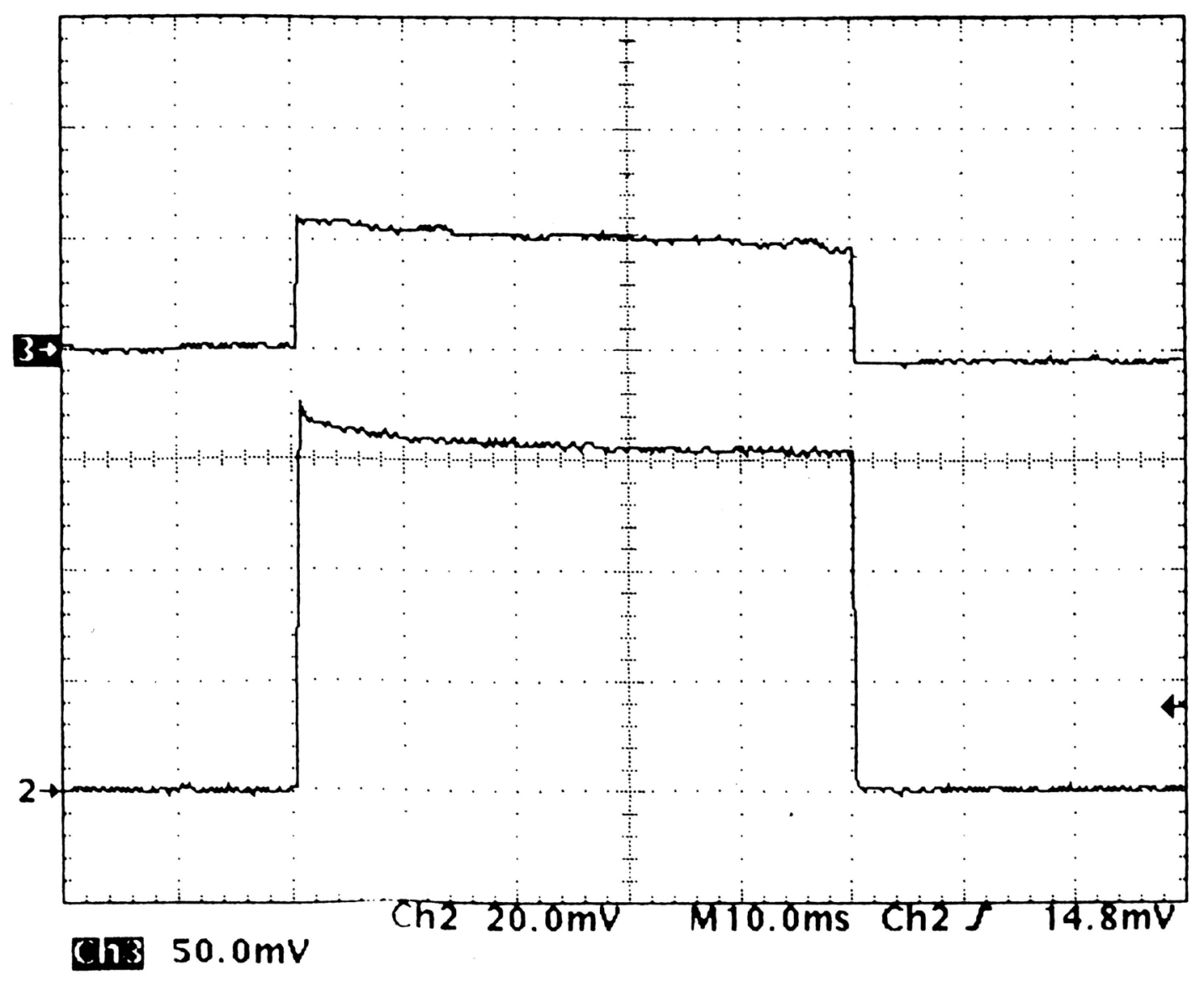

Figure 3 


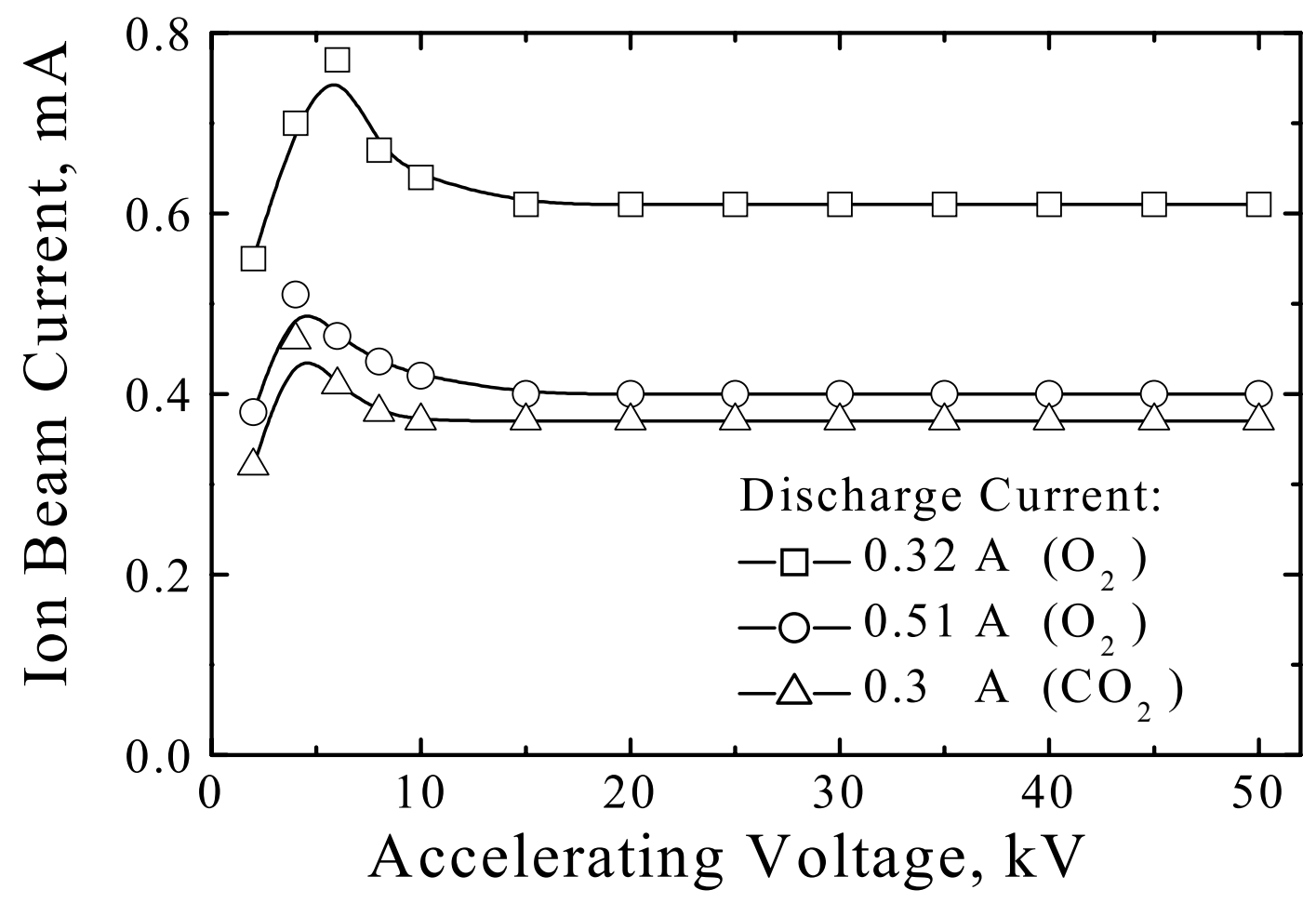

Figure 4 


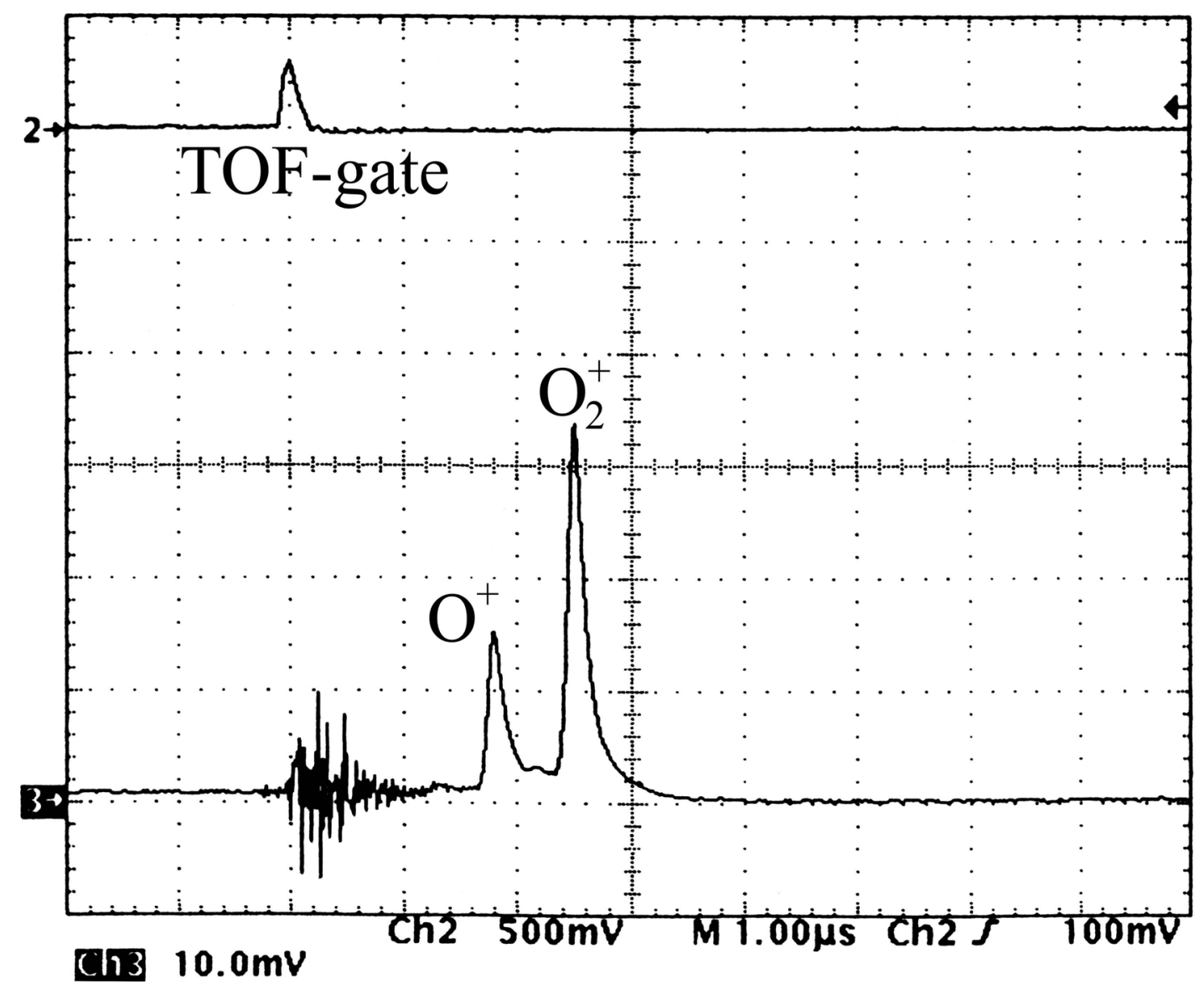

Figure 5 